\title{
Mechanism Analysis of Hydrogen Blisters on the Surface of a Gas Tank and Research on Its Preventive Measures
}

\author{
Yajuan Feng ${ }^{1, a,{ }^{*}}$, Hehui Wang ${ }^{1, b}$, Feng Hou ${ }^{1, c}$, Hu Chen ${ }^{2, d}$, Pingqing $\operatorname{Tan}^{3, \mathrm{e}}$ \\ ${ }^{1}$ School of Mechanical and Power Engineering, East China University of Science and Technology, \\ Shanghai 200237, China; \\ ${ }^{2}$ Ningbo Special Equipment Inspection Center, 315048, China; \\ ${ }^{3}$ Sinopec Zhenhai Refining \& Chemical Company, 315221, China \\ afengyajuan0325@163.com, bhhwang@ecust.edu.cn, chou@ecust.edu.cn, \\ ${ }^{d}$ ch_nbtj@163.com, ${ }^{e}$ tanpq.zhlh@sinopec.com
}

Keywords: Hydrogen Blister, Inclusion, Initiating, Preventive measures.

\begin{abstract}
Serious blisters were found on the shell surface of the gas tank which operated in wet H2S environment during a regular inspection. Gas sampling device was used to determine that the gas in the blister is hydrogen. The causes of blisters were analyzed and investigated by means of chemical analysis, metallographic examines, scanning electron microscopy (SEM) and energy dispersive spectroscopy (EDS). The results revealed that the elongated MnS inclusions are the important reason for the hydrogen blisters. The preventive measures of the hydrogen blisters are put forward based on the above study.
\end{abstract}

\section{Introduction}

Hydrogen blisters are the most common material degradation types caused by hydrogen with high pressure, which are very harmful to pressure vessels[ ]. Carbon steel and low alloy steel are exposed to wet H2S environment, hydrogen atoms are produced for corrosion of the steel, a part of which gets absorbed into the steel and hydrogen atoms combine into hydrogen molecules at or near the interface of second phase particles, metallic matrix and non-metallic inclusion[ ] [ ]. The hydrogen molecules caused a pressure build-up and induced micro cracks or blisters. The cracks more often lie parallel to the rolling plane and to the surface of the steel[ ].

There are many affecting factors of the hydrogen blisters and hydrogen induced cracking, including steel composition, microstructure and the number and shape of the non-metallic inclusion[ ].The shell surface of a petrochemical company's gas tank appears serious blisters. In this paper, blisters were analyzed and investigated by means of chemical analysis, metallographic examines, SEM and EDS, and the preventive measures of the hydrogen blisters are put forward.

\section{Macro Analysis}

The shell surface of a gas tank appears serious blisters when an oil refinery was overhauled in September 2013. The technical parameters of the gas tank are listed in Table 1.

Table 1 The parameters of the gas tank

\begin{tabular}{ccccccc}
\hline $\begin{array}{c}\text { Device } \\
\text { Name }\end{array}$ & Materials & Medium & $\begin{array}{c}\text { Diameter } \\
(\mathrm{mm})\end{array}$ & $\begin{array}{c}\text { Thickness } \\
(\mathrm{mm})\end{array}$ & $\begin{array}{c}\text { Operating } \\
\text { Pressure } \\
(\mathrm{MPa})\end{array}$ & $\begin{array}{c}\text { Operating } \\
\text { Temperature }\left({ }^{\circ} \mathrm{C}\right)\end{array}$ \\
\hline Gas Tank & $20 \mathrm{~g}$ & hydrocarbons, $\mathrm{H}_{2} \mathrm{~S}$ & 500 & 8 & 1.86 & 40 \\
\hline
\end{tabular}

According to the equipment material components supplied by the manufacturers, the chemical composition of the gas tank meets the requirements of the relevant design standard.

As shown in Fig.1, the blisters which have different sizes and drum convex degree are distributed in the tube wall. All blisters defects occurred in the cylinder of $360^{\circ}$ circumference. Some blisters have led to cracks under the gas pressure. And the depth range of blisters is large and there may be a 
lot of internal delamination defects. These are dangerous potentially to the safe operation of the equipment.

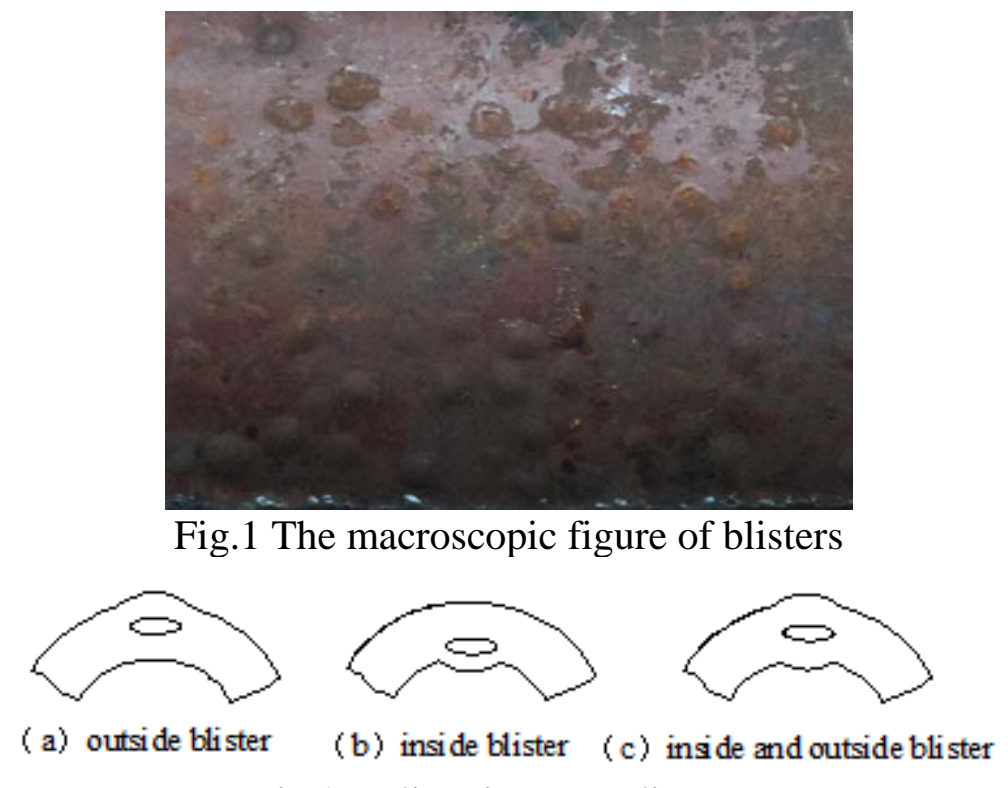

Fig.2 Blistering type diagram

As shown in Fig.2, there are three types of blisters which are called outside blisters, inside blisters and combination of the both respectively. When hydrogen blisters defects are generated during operation, the pressure vessels are usually divided into two sections along the thickness direction, thus gas cavity will be formed between them.

\section{Micro Analysis}

Gas Properties Determined in the Blister Cavity. Gas sampling device was used to determine the gas properties in the blisters, the results showed that the gas was hydrogen. Because the blisters are caused by hydrogen, the hydrogen damage occurred on the hydrogen blisters areas. The material failure degree depends on the process of the hydrogen damage. Therefore, the failure analysis of the hydrogen blisters must be carried on.

The Fracture Test of the Blister.Fig.3 shows the section morphology of the hydrogen blister which is cut along the center of the hydrogen blister. By measurement, the convex thickness of hydrogen blister is 8 to $10 \mathrm{~mm}$ and the diameter is about $20 \mathrm{~mm}$. The blister appears at the outside surface of the shell and the initiated position depends on the non-metallic inclusions on this areas.

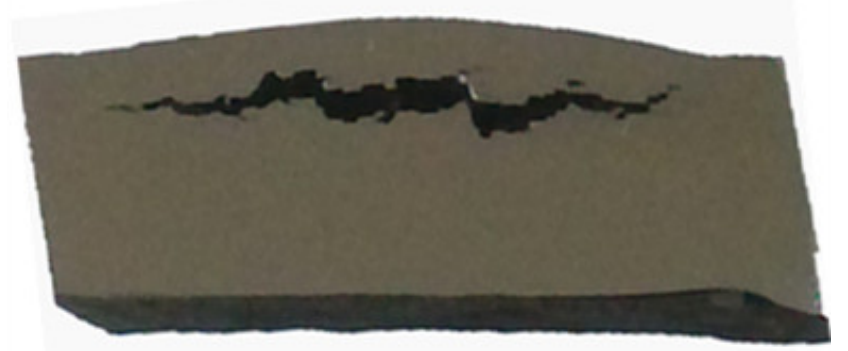

Fig.3 The section morphology of the hydrogen blister

Fig. 4 shows the morphology of the hydrogen blister. The edge of the cracks is jagged and the edge surface is uneven. It's obvious that the plastic deformation was happened in the material and the crack tip was torn off obviously under high pressure. 

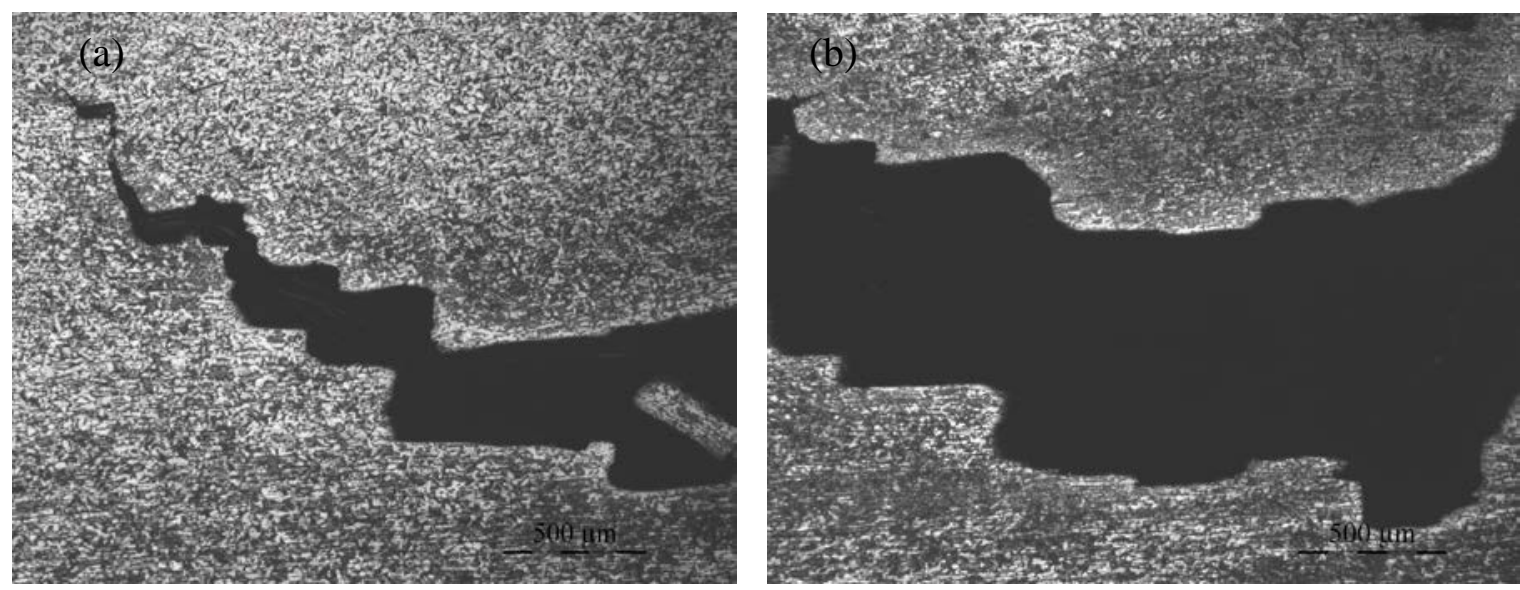

Fig.4 Blister morphology(a)Morphology of crack tip(b) Jagged stratification

The interface of hydrogen blister was torn off artificially.Fig.5 (a) shows the fracture surface of a broken blister where the point $\mathrm{A}$ is the initiation site of the blister and the hydrogen induced crack propagates radially from the initiation site, resulting in a quasi-cleavage fracture on the initiation site. In order to identify the mechanism of the initiation site of the blister, the point A was examined by energy dispersive spectroscopy (EDS) .Fig.5 (b) shows the EDS mapping of the initiation site, and the results showed that the main composition in point $\mathrm{A}$ is element $\mathrm{O}, \mathrm{Fe}, \mathrm{Mn}, \mathrm{S}, \mathrm{P}$ and so on. Then it was found that hydrogen blisters or hydrogen induced cracking could be correlated with the MnS inclusions and $\mathrm{FeO}$ inclusions as well. The defects concentration around the elongated $\mathrm{MnS}$ inclusions far outweighs the matrix, which provide the bases for hydrogen absorption, aggregation and blister formation.
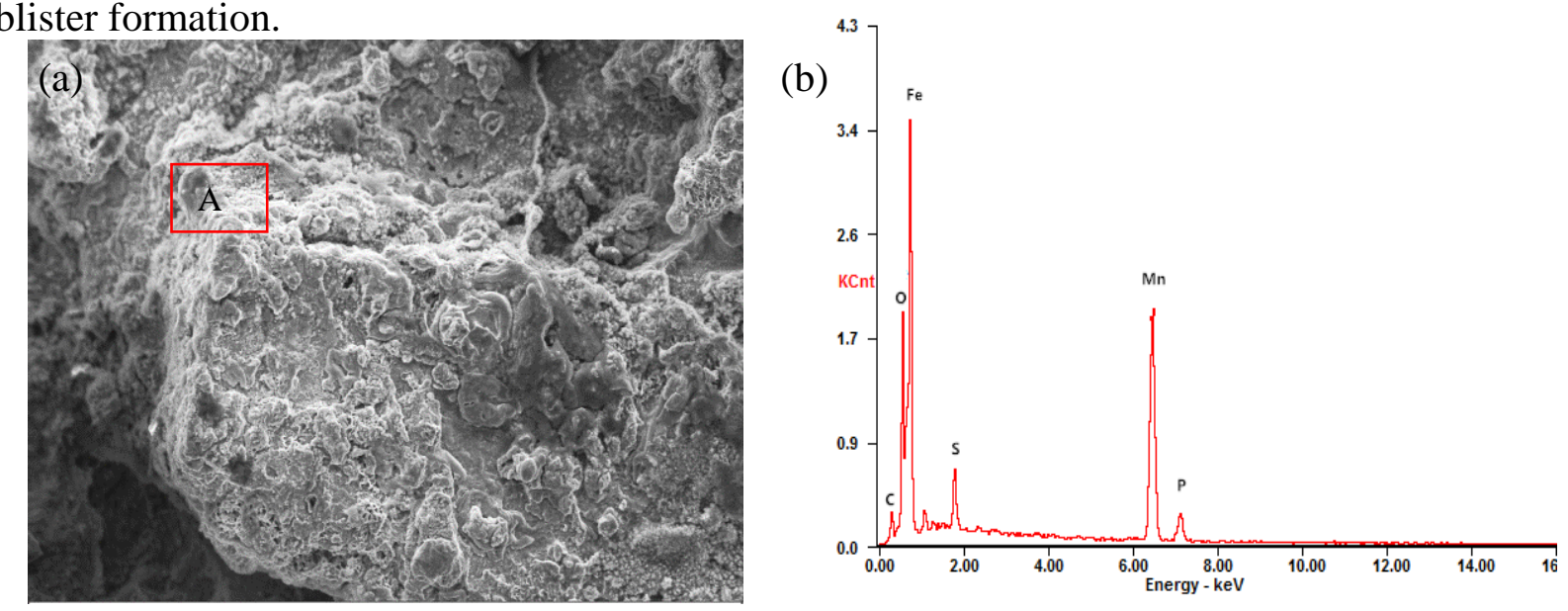

Fig.5 Surface morphology of a hydrogen blister (a) Macroscopically fracture of the blister, point A is the initiation site; (b) EDS of site A

Microstructure Analysis. Sampled from the shell surface, the specimens were ground with silica and mesh number 1200 in a grinding machine, then polished processing, and etched by $4 \%$ nitric acid alcohol solution. After etching, samples were observed under the electron microscope. As shown in Fig.6, the microstructure is ferrite + pearlite, and there are a large number of elongated inclusions. According to the characteristics of the chemical composition of steel and inclusions, the inclusions are MnS. It can be seen from the diagram, the elongated MnS inclusions were distributed along pearlite, the inclusions embedded in the matrix and formed inclusion/matrix interface. These can weaken the bonding force between the metal atoms. 

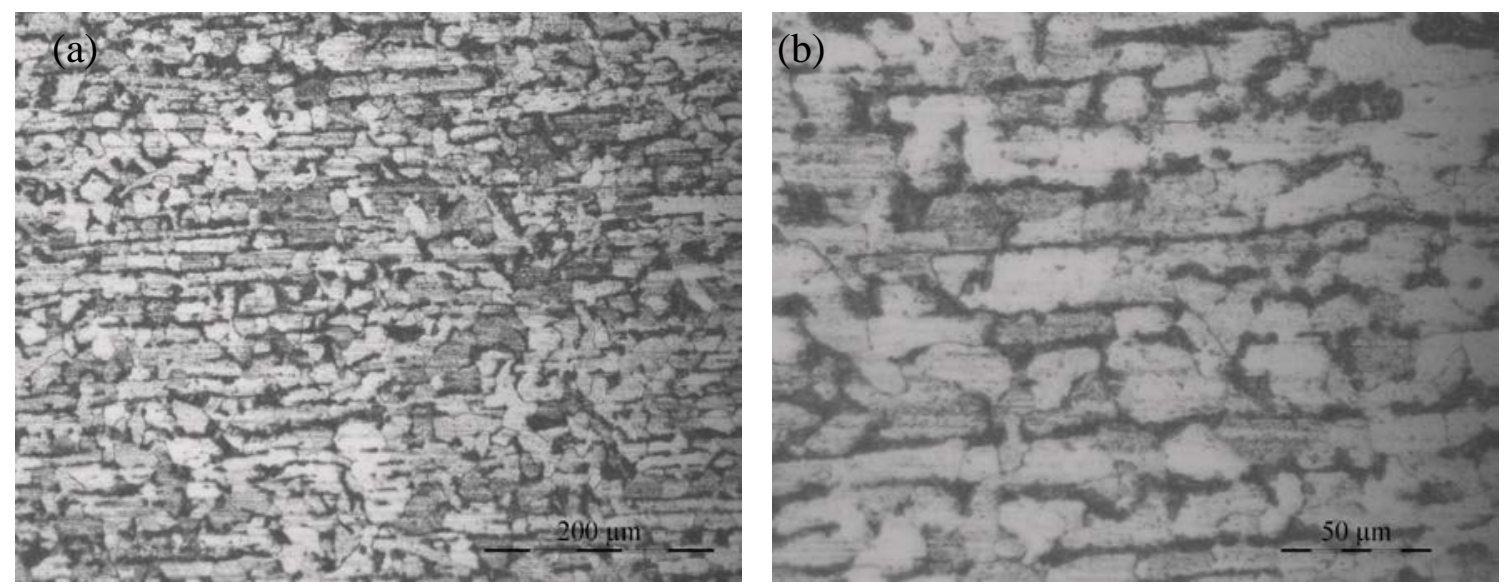

Fig.6 Microstructure (a) The elongated MnS inclusions (200X) (b) Magnification of the MnS inclusions (500X)

\section{Mechanism Analysis of the Blisters}

The presence of $\mathrm{H}_{2} \mathrm{~S}$ and $\mathrm{H}_{2} \mathrm{O}$ in the sour environment induces uniform corrosion and favors $\mathrm{HB}$ on the carbon steel. It has been proposed that the chemical reaction may occur in this environment:

$$
\begin{array}{ll}
\mathrm{H}_{2} \mathrm{~S} \rightarrow \mathrm{H}^{+}+\mathrm{HS}^{-} & \\
\mathrm{L}_{\mathrm{H}^{+}+\mathrm{S}^{2-}} & \\
\text { Anodic reaction: } & \mathrm{Fe} \rightarrow \mathrm{Fe}^{2+}+2 \mathrm{e} \\
\text { Cathodic reaction: } & 2 \mathrm{H}^{+}+2 \mathrm{e} \rightarrow 2 \mathrm{H} \rightarrow \mathrm{H}_{2} \uparrow
\end{array}
$$

There is still a lot of debate on the role of hydrogen in crack and blister formation and several mechanisms have been proposed and discussed. Most of them are based on the internal pressure theory. The interface of second phase and matrix is the trap of hydrogen .The hydrogen concentration in the interface is much higher than that in the matrix. So the atomic size of hydrogen along with iron sulfide porosity allows the former to diffuse into the metallic lattice and be accumulated in voids provided by dislocations and internal faults of metallic microstructure. Atomic hydrogen is recombined to form molecular hydrogen, whose accumulation results in a continuous increase of internal pressure to further bulge in the metal surface, resulting in HB.

\section{Hydrogen Blisters Preventive Measures}

The Causes of Hydrogen Blisters. According to the above comprehensive analysis, there are many causes which induced the hydrogen blister. However, there are mainly two aspects: the steel plate material and the medium. Because the contents of S and $\mathrm{P}$ in the steel exceed standard and there are many MnS inclusions in the steel, which are the main reasons for the formation of the hydrogen blisters. However, hydrogen sulfide and aqueous solution are the medium conditions for forming hydrogen blister.

Preventive Measures of Hydrogen Blister. According to the causes of the hydrogen blisters, there are several measures were put forward to prevent the hydrogen blister:

They are chemical inclusion shape control (Ca, or rare earth treatment) and /or the production of steels with extra low sulphur (lower than $0.002 \%$ ).

Using protective coating on the surface of a container to prevent hydrogen atoms from diffusing into the steel.

\section{Conclusion}

In this paper, blisters on the gas tank in some refinery were analyzed by some experimental means. The obtained conclusions are as follows:

The elongated MnS inclusions are the important reason which led to the hydrogen blisters. 
Hydrogen atoms are released from the corroded surface of steel; they diffuse quickly into the steel through H2S catalysis and agglomerate at the boundaries between inclusions and matrix to form molecular hydrogen. With the pressure going on, a high pressure is generated finally to form hydrogen blister.

Controlling chemical inclusion shape (Ca, or rare earth addition) and extra low sulphur (lower than $0.002 \%$ ), so decrease the content and control the shape of the non-metallic inclusion.

\section{Acknowledgments}

The authors wish to thank the Administration of Quality Supervision, Inspection and Quarantine science and technology plan projects(2014QK172) and Sinopec Zhenhai Refining \& Chemical Company (2014KF002) scientific research project for financial support of this work.

\section{References}

[1] Jun Hu, Fei Liu. Failure analysis based on J-integral values: A case study of hydrogen blistering defect. Engineering Failure Analysis.18(2001)924-932.

[2] C. Zapffe, C. Sims. Hydrogen embrittlement, internal stress and defects in steel. TMS-AIME. 145 (1941) 225-261.

[3] A. S Tetelman, W. D Robertson. The mechanism of hydrogen embrittlement observed in iron-silicon single crystals, Trans. TMS-AIME 224 (1962) 775-783.

[4] D. Perez Escobar,C. Minambres. Internal and surface damage of multiphase steels and pure ironafter electrochemical hydrogen charging, J. Corrosion Science .53 (2011) 3166-3176

[5] E. Villalba, A. Atrens. Hydrogen embrittlement and rock bolt stress corrosion cracking, Eng. Fail Anal. 16 (2009) 164-175. 